\title{
Adaptive Modelling of Multipath Communication Channels Based on the Least Mean Square Algorithm
}

\author{
Dhimas Arief Dharmawan ${ }^{* 1}$ \\ ${ }^{1}$ Department of Electrical Engineering, Faculty of Engineering, Universitas Muhammadiyah Yogyakarta \\ Jalan Brawijaya, Geblangan, Tamantirto, Kasihan, Bantul 55183, Telp (0274) 387656 \\ *Corresponding author, e-mail: dhimasariefdharmawan@umy.ac.id
}

\begin{abstract}
The objective of this research is to design an adaptive model for multipaths communication channels. To evaluate the performance of the model, the authors used two indicators, namely the attainment of a minimum value of error signals in the form of interface and echo on the channel, and Filter Finite Impulse Response (FIR) has the same form of equation as the channel equation used. This adaptation process uses the Least Mean Square (LMS) algorithm. The LMS algorithm is known as a simple algorithm, which is fast computation time and the computation results are quite satisfying. In this study, the authors used Simulink software on Matlab. In general, this research is divided into three stages, namely modeling multipaths communication channels as an adaptive system, designing an adaptive system model on Simulink, and designing a simulation of 250 iterations. After obtaining the simulation results in the form of errors and weight of the FIR filter, it is observed that both of the results have met the requirements of the indicators of the success of this study. With 250 iterations used, satisfactory results were obtained in the form of the fulfillment of all indicators of the success of this study.
\end{abstract}

Keywords: adaptive systems, FIR, error, LMS, multipaths modelling

\section{Introduction}

The term multipaths communication channel is commonly used in the field of telecommunications engineering. This term refers to a communication system that uses more than one channel to transmit information from the sender to the recipient. Multipaths communication channels are considered effective in transmitting information from the sender to the recipient. However, this system has several weaknesses including interference and echo problems.

One way that can be used to minimize interference and echo is to use adaptive system modeling. In this case, the adaptive system is used to identify the shape of the canal plant used.

Adaptive systems have a variety of algorithm choices that can be used, including Steepest Descent, Least Mean Square (LMS), and Recursive
Least Squares (RLS). The algorithm that I use in my research is the LMS algorithm because the LMS algorithm is a simple algorithm. The simplicity lies in the ease of the algorithm to be generated and applied. LMS algorithm is the most efficient computational method in terms of memory, time, and economy in each iteration when compared with the other two algorithms as mentioned above.

\section{Objective}

The objective of this study is to design an adaptive system model that is able to identify the shape of a canal plant with the following indicators of success.

1. The achievement of a minimum error value representing interference and echo and convergence at each Filter Finite Impulse Response (FIR) weight as will be proven in the results and discussion section.

2. Form of equation The FIR filter has the form

Copyright (C) 2017 Universitas Muhammadiyah YogyakartaAll rights reserved 
of an equation with the channel equation used. So it can be said that the process of identifying the shape of the channel was successfully carried out.

Thus, the authors hope this research can help telecommunications experts in using multipaths communication channels.

\section{Theoretical Basis}

\section{A. Scattered Spectrum Theory}

In the Scattered Spectrum Theory, each bit of information both 1 and 0 bits is transmitted as a string bit. Bit 1 is represented as a 32-bit long string and 0 bits are represented as the remaining bit strings of the same length. To detect the received bits, the receiver correlates to the bit string, which is then made as a 1 or 0 bit based on the maxima correlation.

Bits 1 and 0 are pseudo random numbers that are designed so that they are orthogonal and each autocorrelated with a value other than 0 in lag 0 and 0 for the other lags. The maximum length of a sequential shift register has the characteristics mentioned above and has been widely applied in this matter.

On the sending device, there is a switch that moves with a certain clock and changes to generate pulses in the form of bits 1 or 0 according to the pattern of information represented by these bits. These bits of information then propagate through the channel or transmission medium to the receiving device.

The receiving device also has a clock to generate a series of bits 1 and 0 . This clock has the same speed as the clock speed on the sending device, but there is a delay due to a delay on the channel.

Bits 1 and 0 raised on the receiving device are then correlated with the received noisy signals. If the clock between the sending and receiving devices is matched, one of the cross-correlates will produce an output which is then compared by the comparator. So that the resulting bit output is appropriate

\section{B. Adaptive System Theory}

The word adaptive comes from the English language to adapt which means adjusting to the needs or conditions. In recent years, the term adaptive system refers to adaptive automation.

Adaptive system is a system that can be regulated in performance and / or behavior in a certain way to adjust to changes that occur in the surrounding environment. A simple example of the adoption of an adaptive system is the Automatic Gain Control (AGC) circuit on receiving devices found on radio and television. The function of this circuit is to adjust the sensitivity of the receiving device from the average strength of the received signal.

Basically, the adaptation process is divided into two, namely open loop adaptation and closed loop adaptation. The open loop adaptation process begins with measuring the input or environmental characteristics. Then the measurement results are applied to a formula or computational algorithm. The results of this application are then used to adjust the adaptive system.

On the other hand, the closed loop adaptation process develops automatic experiments with known outputs as parameter settings to optimize system performance. Henceforth, this process can be referred to as an adaptation using performance feedback.

Figure 1 displays the block diagram of the closed loop adaptation process. Input cues are denoted by $\mathrm{x}$ and the desired cues are denoted by d. Error or error value is the difference between the desired signal and the input signal. The error value is then used to adjust the structure of the adaptive system with the ultimate goal of minimizing the error. There are various algorithms that can be used in managing the adaptive system structure, including Steepest Descent, Least Mean Square (LMS), and Recursive Least Squares (RLS).

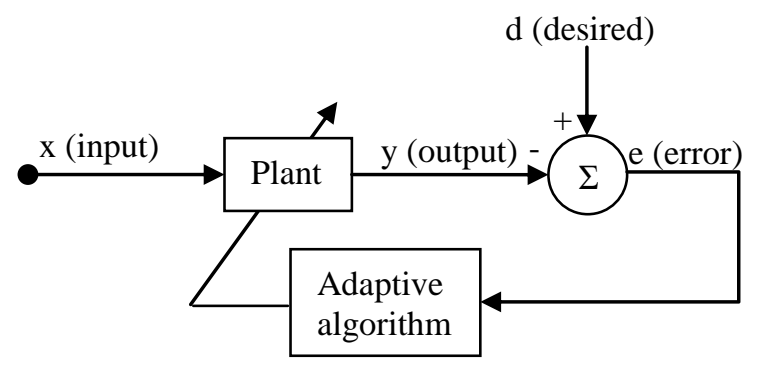

Figure 1. Block diagram of the Closed Loop Adaptation Process

\section{Least Mean Square (LMS) Algorithm}

The Least Mean Square (LMS) algorithm was invented by Widrow and Hoff in 1960. The LMS algorithm is an important member of the stochastic gradient algorithm family. The term stochastic gradient is intended to distinguish the LMS algorithm from the Steepest Descent method which uses deterministic gradients in the recursive calculation of Wiener Filters for stochastic input. 
The main advantage of the LMS algorithm is its simplicity that does not require the measurement of the correlation function between input cues, weights, and desired cues. In addition, the LMS algorithm also does not require an inverse matrix. This is the simplicity of the LMS algorithm which makes it more widely used in the adaptation process compared to other algorithms.

LMS algorithm is an adaptive linear filtering algorithm which consists of two basic processes, as follows.

1. A filtering process that involves calculating the output of a transversal filter produced by a set of inputs and producing an estimation error by comparing this output to the desired response.

2. Adaptive processes that involve automatic adjustment of filter weights according to estimation errors.

Figure 2 explains the representation of the Least Mean Square (LMS) algorithm.

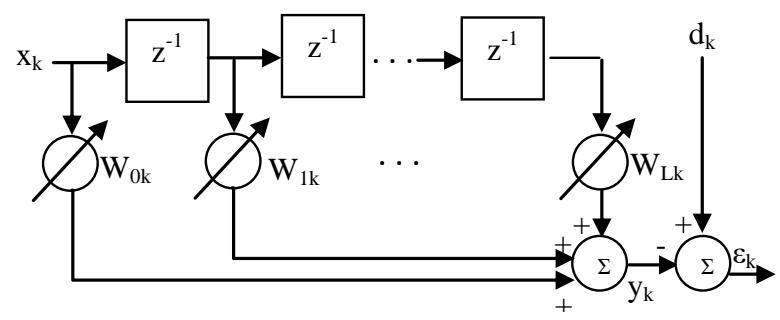

Figure 2. Representation of LMS Algorithm as a Transverse Filter

LMS algorithm is built from the Steepest Descent algorithm which has the following equation.

$$
\begin{gathered}
\nabla_{w} J(n)=-2 p+2 R w(n) \\
\nabla_{W} J(n)=-2 p+2 R w(n) \\
g=\nabla_{w} J(n)
\end{gathered}
$$

If you have obtained the exact vector gradient value $\nabla J(n)$ at each iteration and the $\mu$ value has been determined, you can obtain the optimal weight value from the FIR Filter through the Wiener-Hoff equation. However, it is difficult to obtain the vector value of $\nabla J(n)$. Because the value of the matrix $\mathrm{R}$ is not known, the results of the autocorrelation of the input signal and the matrix $\mathrm{p}$, which are the result of the correlation of the input signal and the desired signal. As a result, the estimated gradient vector $\nabla \mathbf{J}(\mathrm{n})$ is performed.
To get the estimated gradient vector $\nabla \mathbf{J}(\mathrm{n})$, an estimation of the matrix $\mathrm{R}$ and $\mathrm{p}$ is performed. The equation to calculate the estimated values for the matrix $\mathrm{R}$ and $\mathrm{p}$ is as follows.

$$
\begin{aligned}
& \hat{R}=u(n) u^{H}(n) \\
& \hat{p}=u(n) d^{*}(n)
\end{aligned}
$$

With the estimated values of the matrix $R$ and $p$ above, the vector gradient equation $\nabla J$ (n) can be constructed as follows.

$$
\begin{gathered}
\hat{\nabla}_{\hat{w}} J(n)=-2 u(n) d^{*}(n)+2 u(n) u^{H} \hat{w}(n) \\
\hat{w}(n+1)=\hat{w}(n)+\mu u(n)\left[d^{*}(n)-u^{H} \hat{w}(n)\right]
\end{gathered}
$$

From the three equations above, new equations for filter output, error estimation values, and filter weights can be determined as follows.

Filter Output: $y(n)=\widehat{w}^{H}(n) u(n)$

Error: $e(n)=d(n)-y(n)$

Filter weight: $\hat{w}(n+1)=\widehat{w}(n)+\mu u(n) e^{*}(n)$

The weights of the filter in (11) are updated, such that the error in (10) is minimized. The procedure for updating the weights are depicted in Figure 3.

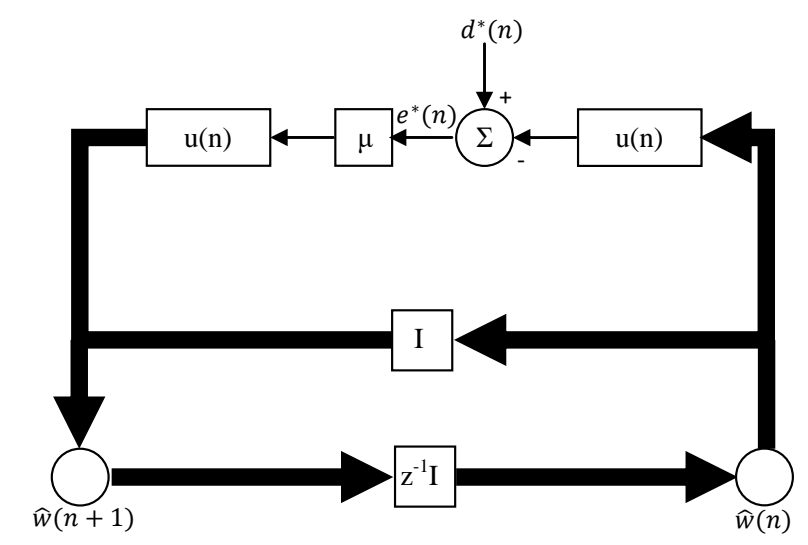

Figure 3. The process of changing filter weights on the LMS algorithm

\section{Methodology}

This research can be divided into three stages, namely modeling multipaths communication channels as an adaptive system, designing an 
adaptive system model in MATLAB, and simulating an illuminated model, as illustrated in Figure 4 below.

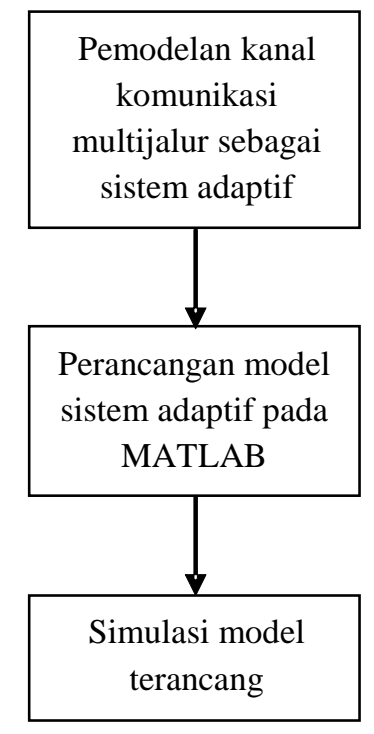

Figure 4. Flow Chart of Research

\section{A. Multipaths Communication Channel Modeling} as an Adaptive System

The first step that must be done in this research is to model multipaths communication channels into an adaptive system model. This modeling is illustrated in Figure 5

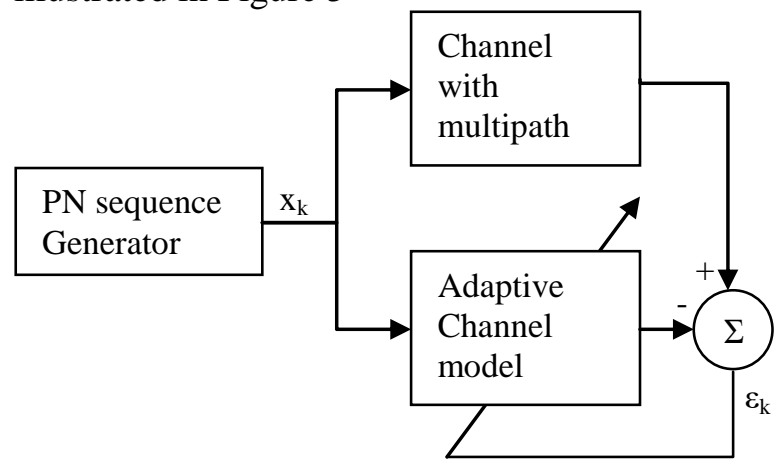

Figure 5. Modeling of Multipaths Communication channels into Adaptive Systems

Enter PN sequence generator: 1110100

The form of channel with multipath is defined by the following equation

$H(z)=1-0,5 z^{-1}+0,25 z^{-2}+0,4 z^{-7}-0,2 z^{-9}+0,12 z^{-9}$
The second phase of this research is designing an adaptive system model obtained from the first stage using the Simulink application on MATLAB. The design results are illustrated in Figure 6.

\section{Designed Model Simulation}

After the adaptive system model is designed, the last stage of this research is to simulate the design. In this case the author simulates the design as many as 250 iterations.

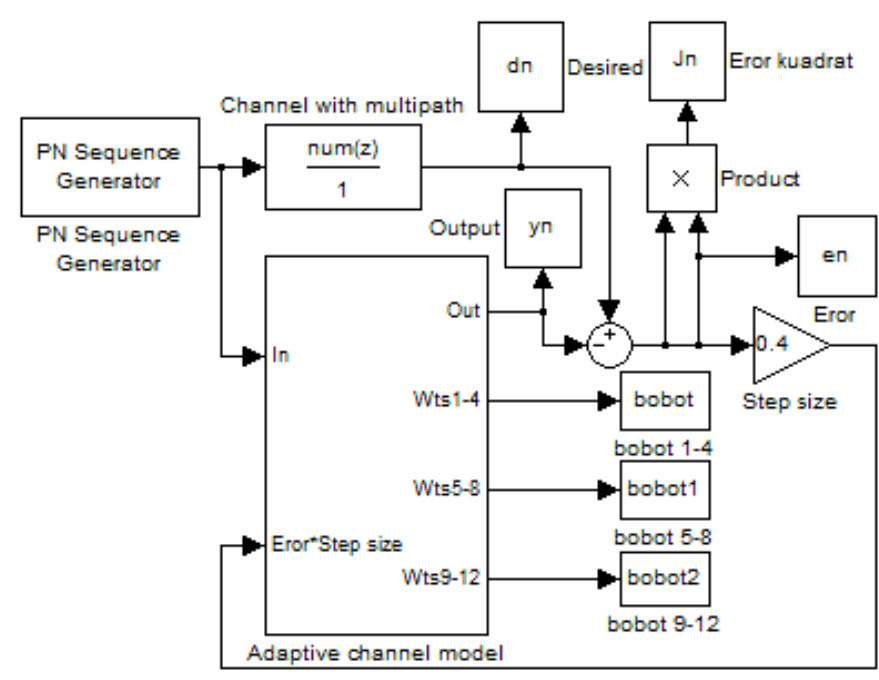

Figure 6. Adaptive System Model Design on MATLAB Simulink

\section{Results and Discussion}

From the simulation, several results will be obtained, namely the desired signal, filter output signal, error, and filter weight. These results are each presented in the form of a two-dimensional graph. The horizontal axis shows the number of iterations and the vertical axis shows the parameters measured.

The simulation results in the form of the desired signal are shown in Figure 7. These signals are obtained from the results of the convolution between the input signal and the channel with multipath equation. Desired cues can be represented by the following equation (Oppenheim, A. V. 2000).

$$
d(n)=u(n) * h(n)
$$

\section{B. Model Design in Matlab}


$\mathrm{u}$ (n) represents the input signal. $\mathrm{h}(\mathrm{n})$ is the result of inverse $\mathrm{z}$ transformation of the channel with multipath equation.

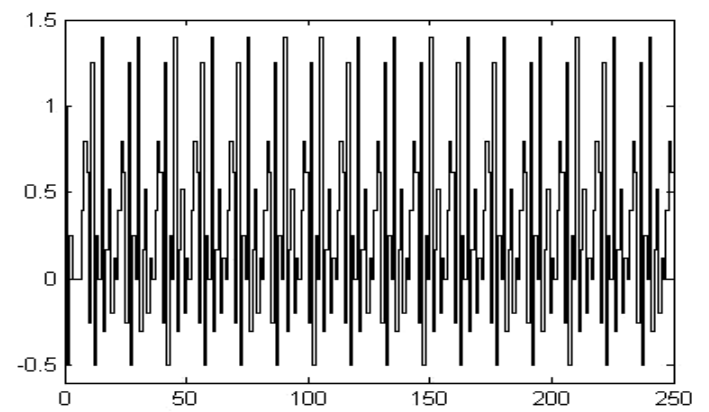

Figure 7. Simulation Results in the Form of Desired Cues

The next simulation results of the filter output are shown in Figure 8. This signal is obtained from the convolution results between the input signal and the FIR Filter equation. The filter output signal is shown by the following equation (Oppenheim, A. V. 2000).

$$
y(n)=u(n) * \widehat{h}(n)
$$

$\mathrm{u}$ (n) represents the input signal. $\mathrm{h}^{\wedge}$ ( $\mathrm{n}$ ) is an estimation of the channel with multipath equation. Desired cues and filter output cues will be taken the difference to obtain the error value of the adaptation process.

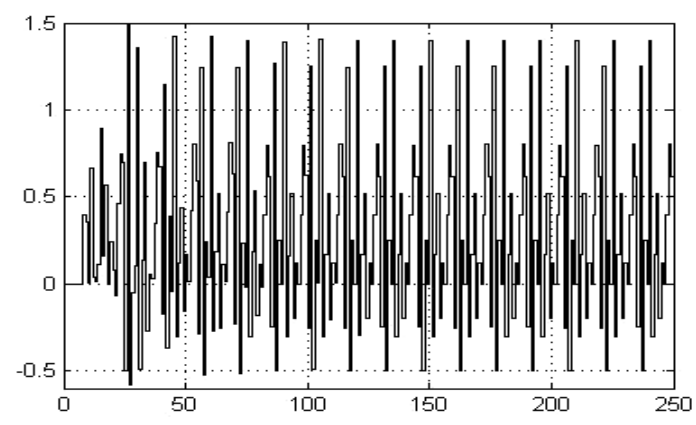

Figure 8. Simulation Results in the Form of Filter Output Signs

The next simulation results in the form of adaptation process errors are shown in Figure 9. Next, the adaptation process error values are detailed in Table 1. The adaptation process errors are obtained from Equation (10).

From Figure 9 and Table I, it appears that the error in the adaptation process is reduced during the iteration process. Error values close to zero are found in the 160th iteration. An iteration of more than 160 is done to make the reader more convincing.

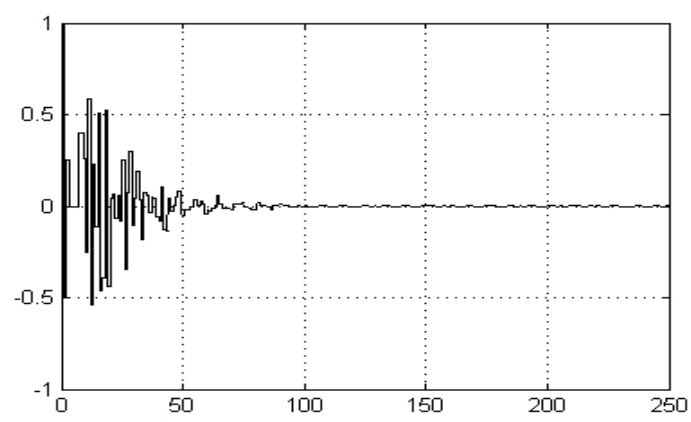

Figure 9. Simulation Results in the Form of Error

The next simulation results in the form of an adaptation process quadratic error are shown in Figure 10. Furthermore, the system quadratic error values are detailed in Table 1 . The system quadratic error is obtained from the following equation (Haykin, S. 2002).

$$
l(n)=E\left\{|e(n)|^{2}\right\}=E\left\{e(n) e^{2}(n)\right\}
$$

From Figure 10 and Table I, it appears that the system square error decreases rapidly during the iteration process. The system square error value close to zero is found in the 90th iteration. The zero square error value obtained quickly shows that iteration is satisfactory. Iteration with a number of more than 90 is done to be more convincing to the reader.

TABLE I. ERRORS AND ERRORS QUADRATES OF

\begin{tabular}{|c|c|c|}
\hline $\begin{array}{l}\text { Number of } \\
\text { iterations }\end{array}$ & Galat Number & $\begin{array}{c}\text { Quadratic error } \\
\text { value }\end{array}$ \\
\hline 1 & 1 & 1 \\
\hline 10 & 0,26 & 0,0676 \\
\hline 20 & 0,04305 & $1,8534 \times 10^{-3}$ \\
\hline 30 & 0,0409 & $1,6776 \times 10^{-3}$ \\
\hline 40 & $-0,078$ & $6,0771 \times 10^{-3}$ \\
\hline 50 & $-0,0526$ & $2,7749 \times 10^{-3}$ \\
\hline 60 & $-0,0191$ & $3,6551 \times 10^{-4}$ \\
\hline 70 & $-0,022$ & $4,0892 \times 10^{-4}$ \\
\hline 80 & $9,2525 \times 10^{-3}$ & $2,41 \times 10^{-4}$ \\
\hline 90 & $8,1788 \times 10^{-3}$ & 0 \\
\hline 100 & $4,7524 \times 10^{-3}$ & 0 \\
\hline 110 & $-1,8135 \times 10^{-3}$ & 0 \\
\hline 120 & $-1,7394 \times 10^{-3}$ & 0 \\
\hline 130 & $-8,7314 \times 10^{-4}$ & 0 \\
\hline 140 & $3,93 \times 10^{-4}$ & 0 \\
\hline 150 & $4,6464 \times 10^{-4}$ & 0 \\
\hline 160 & $2,0416 \times 10^{-4}$ & 0 \\
\hline 170 & 0 & 0 \\
\hline 180 & 0 & 0 \\
\hline
\end{tabular}
THE ADAPTATION PROCESS

Journal of Electrical Technology UMY, Vol. 1, No. 4 


\begin{tabular}{|l|l|l|}
\hline 190 & 0 & 0 \\
\hline 200 & 0 & 0 \\
\hline 210 & 0 & 0 \\
\hline 220 & 0 & 0 \\
\hline 230 & 0 & 0 \\
\hline 240 & 0 & 0 \\
\hline 250 & 0 & 0 \\
\hline
\end{tabular}

Figures 11, 12 and 13 show the weight graph of the FIR filter used. Figure 11 displays the first weights up to the fourth weight of the FIR Filter, Figure 12 displays the fifth weight to the eighth weight of the FIR Filter and Figure 13 displays the ninth to the twelfth weight of the FIR Filter. This weight value is obtained from Equation (11).

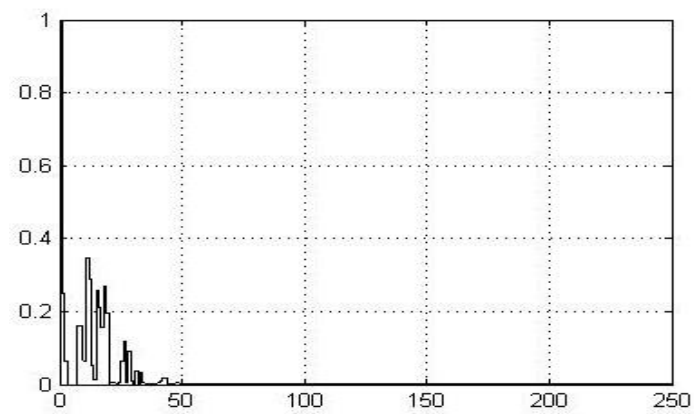

Figure 10. Simulation Results in the Form of Quadratic Errors

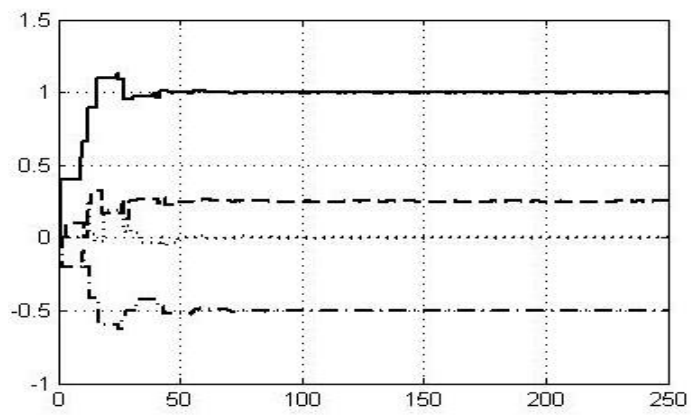

Figure 11. Simulation Results in the Form of the First to Fourth Filter Weights

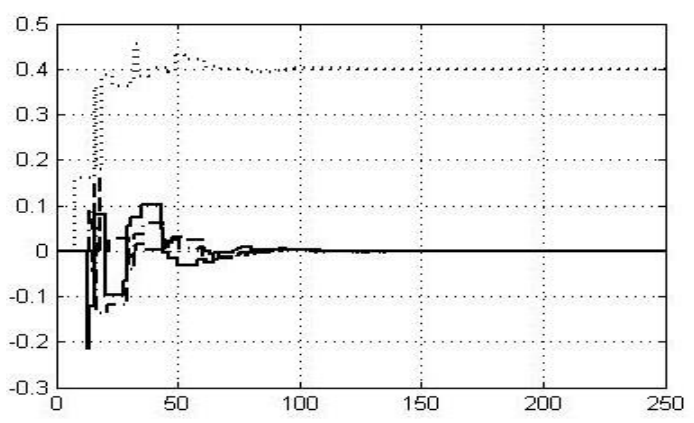

Figure 12. Simulation Results in the Form of Weights Fifth to Eight Filters

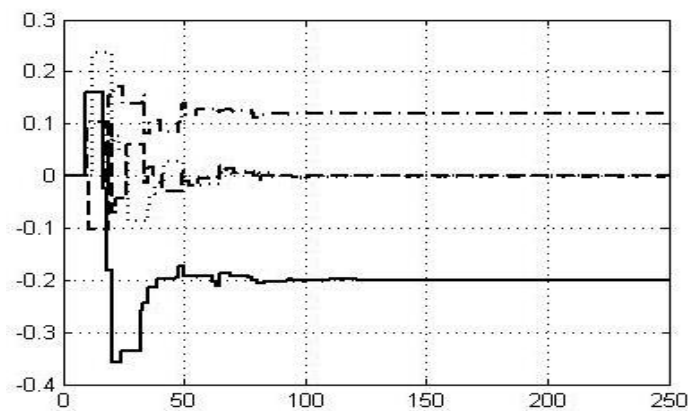

Figure 13. Simulation Results in the Form of Weights Ninth to Twelve Filters

In the graph there are twelve filter weights which indicate the length of the FIR filter used. Each weight changes to the convergent point. This change is influenced by the product of the error and $\mu$ times. The convergence indicator for each weight is shown by each weight value which has not changed back to a certain number of iterations. It appears that each weight is convergent at the hundredth iteration. The convergent weight values are shown in Table II.

TABLE II. WEIGHT CONVERGENT FILTERS

\begin{tabular}{|c|c|c|}
\hline Line Type & $\begin{array}{l}\text { Weight } \\
\text { Filter } \mathrm{n}\end{array}$ & $\begin{array}{c}\text { Weight Filter } \\
\text { Value } \mathrm{n}\end{array}$ \\
\hline 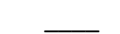 & 1 & 1 \\
\hline -.-.-.- & 2 & $-0,5$ \\
\hline ------ & 3 & 0,25 \\
\hline$\ldots \ldots$. & 4 & 0 \\
\hline$=$ & 5 & 0 \\
\hline -..-.- & 6 & 0 \\
\hline ------ & 7 & 0 \\
\hline$\ldots \ldots$. & 8 & 0,4 \\
\hline$\overline{\bar{L}}$ & 9 & $-0,2$ \\
\hline.$-^{-}$ & 10 & 0,12 \\
\hline ------ & 11 & 0 \\
\hline$\ldots \ldots$. & 12 & 0 \\
\hline
\end{tabular}

From the error results and filter weights obtained, a relationship can be drawn that when all filter weight values are converging, the adaptation process error values are close to zero. The error value that has approached zero and the convergence achieved at each weight of the FIR filter is one indicator of the success of this study.

From Table II, we get the final weight value of the filter which can be used to define the filter equation as follows.

$H(z)=1-0,5 z^{-1}+0,25 z^{-2}+0,4 z^{-7}-0,2 z^{-8}+0,12 z^{-9}$

The form of Equation (16) is the same as the form of Equation (12) which is the Channel With Multipath Equation. This makes achieving one of the indicators of success, the FIR filter has the same form of equation as the channel equation used. 


\section{Conclusion}

From the research conducted above, it can be concluded that this research was successful in accordance with the indicators on the purpose of this research, which are as follows.

1. The achievement of a minimum error value representing interference and echo and convergence at each weight of the FIR Filter as proven in the results and discussion section.

2. The shape of the equation The FIR filter has the form of the channel equation used, so it can be said that the process of identifying the shape of the channel was successfully carried out.

\section{References}

[1] B. Widrow, and S. D. Stearns, "Adaptive Signal Processing". New Jersey: Prentice Hall PTR. 1985.

[2] S. Haykin, "Adaptive Filter Theory 4rd ed". New Jersey: Prentice Hall PTR. 2002.

[3] A. V. Oppenheim, and A. S. Willsky, "Sinyal dan Sistem Edisi Kedua". Jakarta: Erlangga. 2000. 